\title{
Editorial
}

\section{Molecular Targeted Therapy for Breast Cancer}

\author{
Breast Cancer 14:131, 2007.
}

Key words: Molecular targeted therapy, Breast cancer
Yoshinori lto

Department of Medical Oncology, Cancer Instifute Hospital, Japanese Foundation for Cancer Research, Japan.
Cytotoxic agents such as anthracycline or taxane had played a central role in the systemic treatment of breast cancer. Significantly improved survival using cytotoxic agents have become the standard for operable breast cancer. In earlier development cycles, anticancer agent which have activity against all kinds of cancer were desired. For instance, doxorubicin, which was derived by screening for activity in the mice leukemia cell line L1210, has a broad spectrum of efficacy for cancer. However, doxorubicin is inevitably accompanied by intolerable toxicities such as emesis, alopecia, mucositis and myelosuppression. The use of multiple active drugs in combination, either concurrently or sequentially, may improve treatment efficacy. But, it is difficult to test multiple combined cytotoxic drugs due to their toxicities. High dose chemotherapy, dose-dense chemotherapy and metronomic therapy have all attempted to overcome this hurdle (Table 1). Unfortunately, the usefulness of these modified chemotherapies has not been fully established. In contrast, molecular targeted agents can be safely combined with other agents. Some emerging molecular targeted agents with relatively narrow spectrum of activity may overcome the limitations of cytotoxic drugs. Molecular targeted agents may help selected patients, e.g. the monoclonal antibody trastuzumab benefits patients with HER2-overexpressing breast cancer conferring significantly prolonged survival with limited mild toxicities. Selective estrogen receptor modulators or aromatase inhibitors, which target a growth pathway dependent on estrogen receptors, are also a standard systemic treatments. Hormonal agents, which target cancer cellular mole-
Table 1. Types of Systemic Therapy

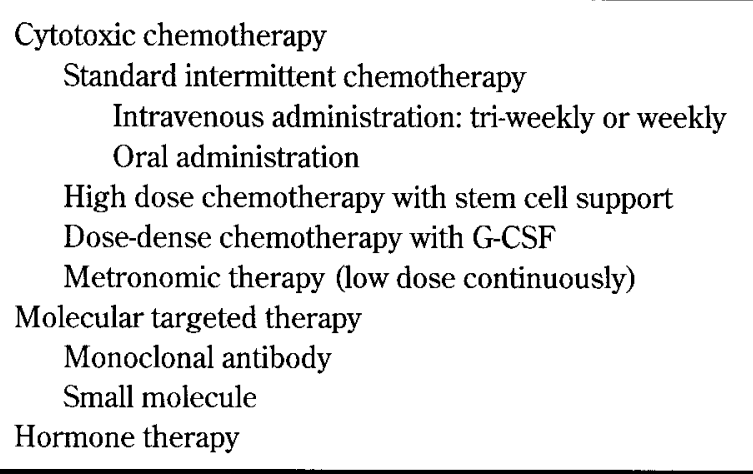

cules, are another molecular targeted agents with significant activity and low toxicities.

The key point is how to identify patients populations who will benefit from molecular targeted agents. The goal of any treatment is maximal activity with minimal toxicity. Hormone therapy with predictive marker for hormone receptors has been successful. Investigation of biomarkers that predict clinical response will make such treatments possible. Translational research should be conducted in clinical trials for developing molecular targeted agents. Novel agents would be developed corresponding to the molecular predictive markers.

The current issue surveys ongoing research into molecular targeted agents for breast cancer. The articles focus the emerging drugs, including monoclonal antibodies or small-molecule inhibitors of signal transduction, especially trastuzumab, lapatinib or bevacizumab. 